Carlos Juarez Velasco

\title{
Metodologia para o Cálculo do Valor do Serviço e da Remuneração dos Agentes Fornecedores de Reservas Operativas
}

TESE DE Doutorado

Departamento de Engenharia Elétrica Programa de Pós-Graduação em Engenharia Elétrica 


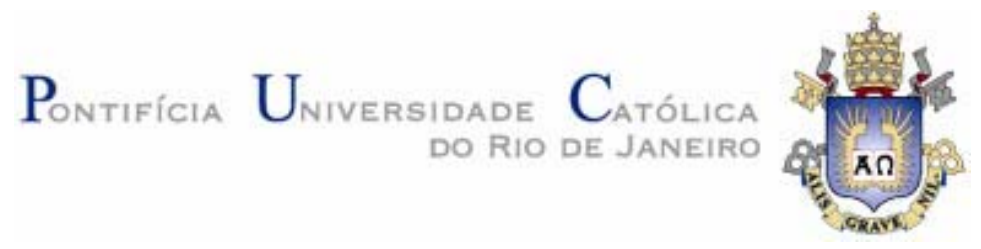

Carlos Juarez Velasco

\section{Metodologia para o Cálculo do Valor do Serviço e da Remuneração dos Agentes Fornecedores de Reservas Operativas}

Tese apresentada como requisito parcial para obtenção do título de Doutor pelo Programa de Pós-Graduação em Engenharia Elétrica da PUC-Rio.

Orientador: Ricardo Prada

Rio de Janeiro, julho de 2004 
Todos os direitos reservados. É proibida a reprodução total ou parcial do trabalho sem autorização da universidade, do autor e do orientador.

Carlos Juarez Velasco

Professor na Faculdade de Engenharia da Universidade Federal de Juiz de Fora desde 1977 graduou-se em Engenharia Elétrica pela Universidade Federal de Juiz de Fora em 1975; mestre em engenharia elétrica pela COPPE Universidade Federal do Rio de Janeiro em 1981.

Ficha Catalográfica

Velasco, Carlos Juarez

Metodologia para o cálculo do valor do serviço e da remuneração dos agentes fornecedores de reservas operativas / Carlos Juarez Velasco ; orientador: Ricardo Prada. - Rio de Janeiro : PUC, Departamento de Engenharia Elétrica 2004.

340 f. : il. ; $30 \mathrm{~cm}$

Tese (doutorado) - Pontifícia Universidade Católica do Rio de Janeiro, Departamento de Engenharia Elétrica.

Inclui referências bibliográficas.

1. Engenharia elétrica - Teses. 2. Serviços ancilares 3. Reserva de potência operativa. 4. Regulação de freqüência. 5. Pagamento por capacidade. 6. Custos. 7. Confiabilidade. 8. Valor de serviço. I. Prada, Ricardo. II. Pontifícia Universidade Católica do Rio de Janeiro. Departamento de Engenharia Elétrica. III. Título.

CDD: 621.3 


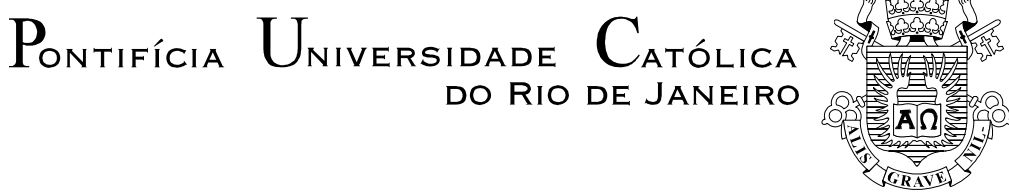

Carlos Juarez Velasco

\author{
Metodologia para o Cálculo do \\ Valor do Serviço e da Remuneração \\ dos Agentes Fornecedores \\ de Reservas Operativas
}

Tese apresentada como requisito parcial para obtenção do grau de Doutor pelo Programa de Pós-Graduação em Engenharia Elétrica do Departamento de Engenharia Elétrica do Centro Técnico Científico da PUC-Rio. Aprovada pela Comissão Examinadora abaixo assinada.

\author{
Dr. Ricardo Bernardo Prada \\ Orientador \\ Departamento de Engenharia Elétrica - PUC-Rio
}

Dr. Acácio Magno Ribeiro UFJF

Dr. Luiz Henrique de Souza Aguiar Coutinho

FURNAS

Dr. Albert Cordeiro Geber de Melo CEPEL

Dr. Antonio José Alves Simões Costa

UFSC

Prof. José Eugenio Leal Coordenador Setorial do Centro

Técnico Científico - PUC-Rio

Rio de Janeiro, 19 de julho de 2004 


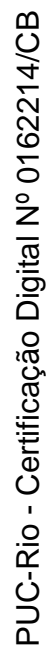

Aos meus pais, Maurício Roberto e Maria de Lourdes

In Memoriam 


\section{Agradecimentos}

Ao professor Ricardo Prada pelo apoio e orientação no desenvolvimento deste trabalho.

À CAPES e à Universidade Federal de Juiz de Fora, pelos suporte financeiro concedido, sem os quais este trabalho não se realizaria.

À Faculdade de Engenharia da UFJF e aos professores do Departamento de Energia Elétrica, por permitirem e incentivarem o meu afastamento para realizar esta pesquisa.

Ao CEPEL ${ }^{1}$, pela cessão das ferramentas computacionais ali desenvolvidas e usadas nesta tese.

À CEMIG e ao ONS pela disponibilização de dados do Sistema Elétrico Brasileiro.

Ao amigo Leonardo Xavier da Silva, pela amizade e pelo apoio na implementação computacional.

À minha esposa Ana Maria e aos meus filhos Bernardo e Maurício pela paciência e compreensão nos períodos de ausência.

A todos os outros companheiros do curso de Pós-Graduação em Engenharia Elétrica, pelo estímulo e pela saudável convivência.

Aos funcionários do Departamento de Engenharia Elétrica da PUC-Rio pela atenção e presteza.

\footnotetext{
1 Parte deste trabalho é resultado do Termo Aditivo No 06/00 ao Convênio entre a Fundação Padre Leonel Franca - FPLF da PUC/Rio e o CEPEL, sobre Serviços Ancilares à Operação de Sistemas Elétricos, de julho 2000 a março 2003.
} 


\section{Resumo}

Velasco, Carlos Juarez. "Metodologia para o Cálculo do Valor do Serviço e da Remuneração dos Agentes Fornecedores de Reservas Operativas". Rio de Janeiro, 2004. 340p. Tese de Doutorado - Departamento de Engenharia Elétrica, Pontifícia Universidade Católica do Rio de Janeiro.

Com a modificação estrutural do setor de produção, transmissão e distribuição de energia elétrica em vários países do mundo, inclusive no Brasil, serviços caracterizados como 'ancilares', que sempre foram oferecidos de forma integrada com o fornecimento de energia elétrica, sem que fossem remunerados especificamente, devem hoje ser tratados e remunerados de forma independente. A necessidade de otimizar custos, em função dos novos ambientes comerciais, muitas vezes competitivos, tem feito com que os agentes geradores vejam o fornecimento de reserva de potência operativa e a regulação de freqüência como novas oportunidades de prestação de serviços. Com isto, novos custos e responsabilidades foram criadas, o que reforça a necessidade de remuneração desses serviços ancilares. Devem-se valorizar os benefícios por eles agregados ao sistema elétrico, representados pelo aumento da confiabilidade e da qualidade da energia. Assim, questões que merecem ser tratadas cuidadosamente são a determinação dos preços a serem pagos pelos consumidores pelos serviços, a determinação correta da participação de cada agente e a necessidade de mecanismos justos de distribuição dos recursos financeiros recebidos por estes serviços entre os agentes que realmente os forneceram. Neste trabalho desenvolve-se uma estrutura de remuneração dos serviços de reservas operativas e regulação de freqüência, baseada no benefício por eles determinado, premiando-se a eficiência e permitindo-se a recuperação dos custos incorridos. Através de estudos de casos com dados reais verifica-se a consistência da proposta de remuneração, comparando os seus resultados com valores já praticados, e analisando o seu impacto sobre as atuais tarifas de energia elétrica.

\section{Palavras - chave}

Serviços ancilares, reserva de potência operativa, regulação de freqüência, pagamentos por capacidade, custos, confiabilidade, valor do serviço. 


\title{
Abstract
}

\begin{abstract}
Velasco, Carlos Juarez. "Methodology for Calculating Service Price and Remuneration for Operating Reserve Supplier Agents". Rio de Janeiro, 2003. 340p. Doctorate Thesis - Electrical Engineering Department, Pontifícia Universidade Católica do Rio de Janeiro.
\end{abstract}

Nowadays, with the structural modification of the electric energy industry around the world, including in Brazil, services characterised as 'ancillary', that always were offered bundled with energy supply, without specific remuneration, should be dealt with independently. The need for cost optimisation, caused by the new commercial environment, sometimes competitive, has made the generating agents to understand operating reserves and frequency regulation as a new opportunity of providing services. Furthermore, new costs and obligations have been created, thus reinforcing the need for these ancillary services to be remunerated. It is necessary to recognise the benefits aggregated to the electric system by ancillary services, represented by improvement of reliability and energy quality. In this way, issues that deserve to be carefully treated are i) the prices to be paid by consumers, ii) the participation of each agent that really provided the service, and iii) the fairness of the mechanism for distribution of the financial resources among the providers. In this work it is developed a structure for the remuneration of operating reserves and frequency regulation, based on the benefit of this services, awarding efficiency and allowing the recuperation of incurred costs. Through study-cases with actual data, it is verified the consistency of the proposal, by comparing its results with values in practice, and by analysing its impact over current electric energy tariffs.

\section{Keywords}

Ancillary services, operating reserve, frequency regulation, capacity payments, costs, reliability, service value. 


\section{Sumário}

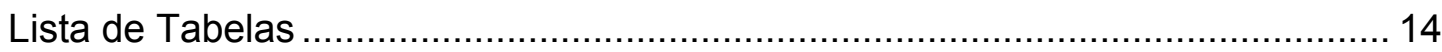

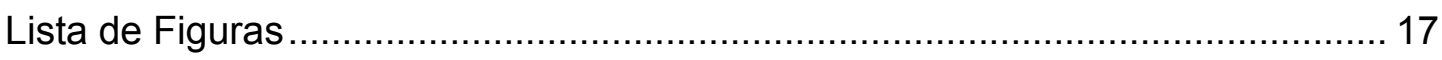

Lista de Abreviaturas, Siglas, Acrônimos e Variáveis .................................... 18

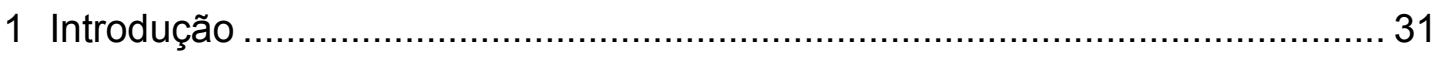

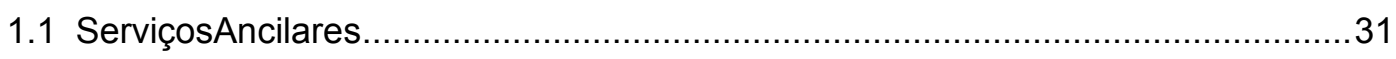

1.1.1 Serviços de Resposta à Freqüência ou Regulação ...................................34

1.1.2 Serviços de Reservas de Potência ...........................................................35

1.1.3 Serviços de Suporte de Potência Reativa e Controle de Tensão ..................37

1.1.4 Serviços de Reinício Autônomo da Operação do Sistema ou Black-Start...38

1.2 A Remuneração dos Serviços Ancilares ........................................................ 38

1.2.1 Modelos de Comercialização .................................................................. 40

1.2.1.1 Modelo Competitivo de Comercialização com Mercado .................40

1.2.1.2 Modelo Competitivo de Comercialização sem Mercado .................44

1.2.1.3 Modelo Não Competitivo de Comercialização ...............................44

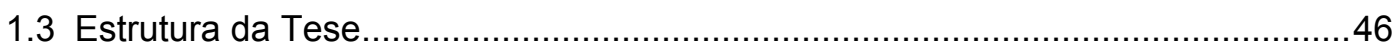

2 Pagamentos por Capacidade ............................................................ 49

2.1 Introdução

2.2 Pagamentos pela Capacidade e Confiabilidade a Longo Prazo ............................51

2.3 Formas de Remuneração da Capacidade ...........................................................54

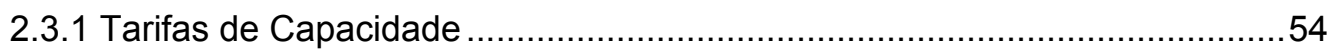

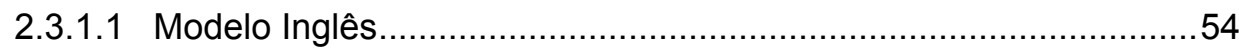

2.3.1.2 Pagamentos por Capacidade na Argentina .................................56

2.3.1.3 Análise Custo x Benefício .....................................................58

2.3.2 Tarifas com Componente de Capacidade ...............................................60

2.4 Experiência Mundial e os Problemas Verificados ...............................................61

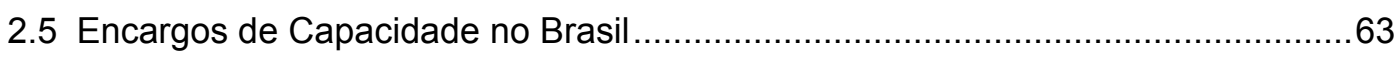

2.5.1 Propostas do Modelo de Reestruturação do Setor Elétrico.........................63

2.5.2 Proposta do Comitê de Revitalização do Modelo do Setor Elétrico ..............66

2.6 Reservas de Planejamento e Reservas Operativas ...........................................68

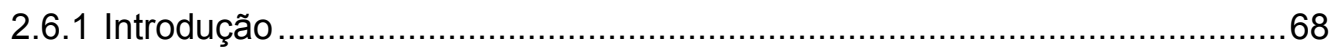


2.6.2 Interação entre as Reservas de Planejamento e as Reservas Operativas ..69

2.6.2.1 Análise do Aspecto Econômico Envolvido ....................................71

2.7 Tarifas de Energia Elétrica e Custos Fixos .......................................................73

2.7.1 Tarifas de Fornecimento de Energia Elétrica na Indústria Brasileira de

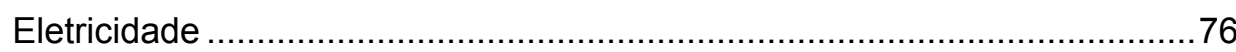

2.7.2 Relacionamento entre Tarifas, Custos Fixos e Confiabilidade .....................78

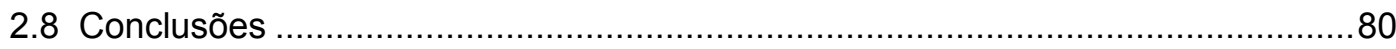

2.8.1 Aplicação dos Encargos de Capacidade .................................................. 80

2.8.2 Utilização dos Encargos de Capacidade da Disponibilidade não Despachada como Remuneração das Reservas de Potência Operativa ......................... 82

3 Custos Incorridos pelos Agentes Prestadores dos Serviços de Reservas Operativas e de Regulação de Freqüência .................................................. 85

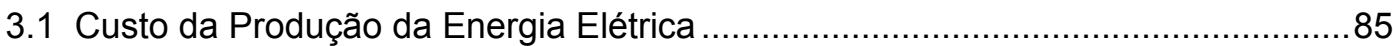

3.1.1 Avaliação dos Custos Envolvidos na Geração da Energia Elétrica...............90

3.2 Custos Relacionados aos Serviços de Reservas Operativas e Regulação de

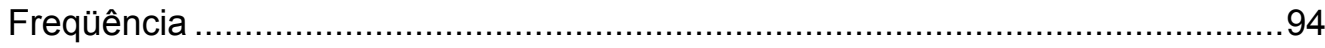

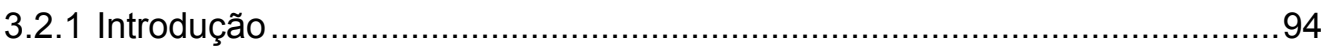

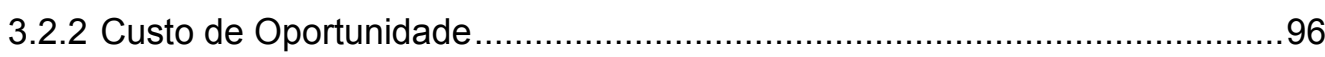

3.2.2.1 Custo de Oportunidade em Ambientes Competitivos ...................105

3.2.3 Influência do Fator de Capacidade ..................................................... 107

3.2.4 Custos Específicos da Reserva de Potência Operativa ............................109

3.2.5 Alocação dos Custos Incorridos pelos Serviços de Reservas Operativas para

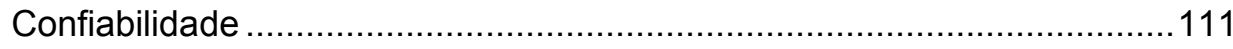

3.2.5.1 Usinas ou Unidades Geradoras Hidráulicas ..............................111

3.2.5.2 Usinas ou Unidades Geradoras Térmicas ..................................118

3.2.6 Alocação dos Custos Incorridos pelos Serviços de Reservas Operativas para Regulação de Freqüência........................................................................122

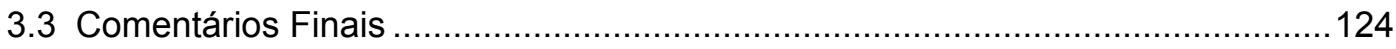

4 Remuneração dos Serviços de Reservas Operativas e de Regulação de

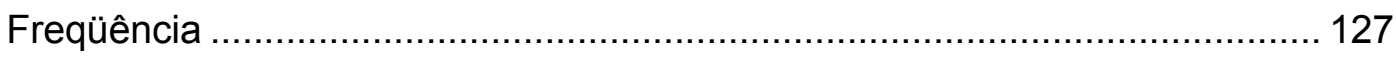

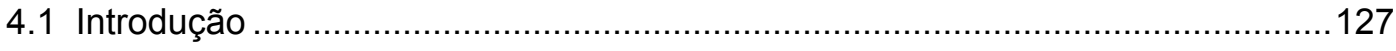

4.2 Eficiência Econômica nas Transações Comerciais Relacionadas às Reservas.. 130

4.3 Definição do Mecanismo de Remuneração dos Serviços...................................133

4.3.1 Justificativa da Remuneração............................................................ 133

4.3.2 Remuneração dos Serviços ................................................................134 
4.3.3 Valor Teto da Remuneração do Benefício dos Serviços de Reservas

Operativas e Regulação de Freqüência .................................................135

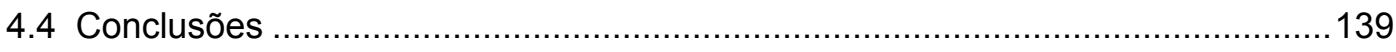

5 Remuneração do Benefício Agregado pelos Agentes Fornecedores de Reservas Operativas para Confiabilidade ............................................. 141

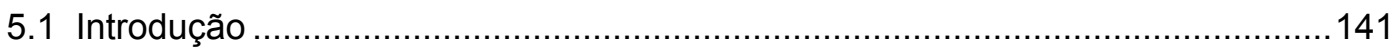

5.2 Mecanismo de Remuneração do Benefício ...................................................... 141

5.2.1 Individualização da Remuneração das Unidades Geradoras Fornecedoras ... de Reservas Operativas para Confiabilidade ........................................ 143

5.2.2 Definição da Remuneração ................................................................ 145

5.3 Cálculo das Probabilidades de Perda de Carga - LOLP ...................................147

5.4 Procedimento para o Cálculo da Remuneração do Benefício Agregado pelas Reservas para Confiabilidade ..................................................................... 150

5.5 Contabilização Mensal da Remuneração da Reserva para Confiabilidade ..........158

5.6 Estudo de Caso 01: Cálculo da Remuneração de Reservas para Confiabilidade..... 158

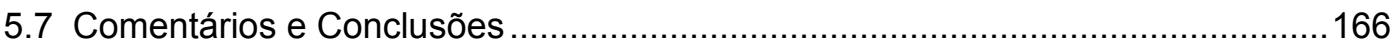

6 Remuneração do Benefício Agregado pelos Agentes Participantes da Regulação da Freqüência ............................................................. 169

6.1 Remuneração do Benefício Agregado pela Reserva Operativa para Regulação de Freqüência 169

6.2 Remuneração do Benefício Agregado pelo Serviço de Regulação de Freqüência ...

6.2.1 Cálculo de um Fator para Avaliação da Eficiência da Regulação de

Freqüência 175

6.2.1.1 Procedimento para o Cálculo do Fator de Eficiência Horária da Regulação de Freqüência 181

6.2.1.2 Aplicação Numérica: Obtenção de Fatores de Eficiência Horária da Regulação Secundária de Freqüência 183

6.2.2 Procedimento para o Cálculo da Remuneração do Benefício Agregado pelo . Serviço de Regulação de Freqüência ....................................................185

6.2.2.1 Procedimento para o Cálculo da Remuneração da Regulação de Freqüência ...................................................................... 188

6.2.2.2 Contabilização Mensal da Remuneração da Regulação de Freqüência 189 
6.3 Estudo de Caso 02: Cálculo da Remuneração da Regulação de Freqüência (Reservas e Serviço) ................................................................................ 190

6.4 Estudo de Caso 03: Remuneração Considerando os Custos.............................. 199 6.4.1 Usinas ou Unidades Geradoras Fornecendo Reservas para Confiabilidade ..

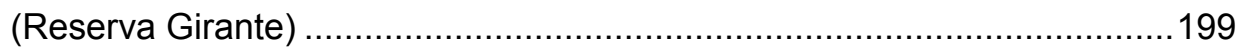

6.4.1.1 Unidade Hidráulica................................................................200

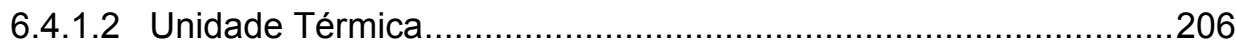

6.4.2 Usinas ou Unidades Geradoras Participantes da Regulação de Freqüência ..

(Reservas e Serviço) .......................................................................211

6.4.2.1 Unidade Hidráulica 'Velha' ...................................................212

6.4.2.2 Unidade Hidráulica 'Nova' .......................................................213

6.4.2.3 Unidade hidráulica - Considerando os Preços dos Contratos Iniciais

6.4.2.4 Unidade hidráulica - Sem Restrição de Geração.........................215

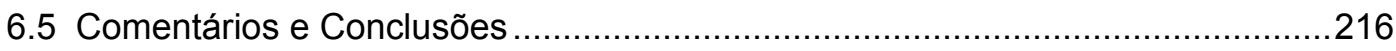

7 Influência do Mecanismo de Realocação de Energia na Remuneração dos Agentes Fornecedores dos Serviços de Reservas Operativas e Regulação de

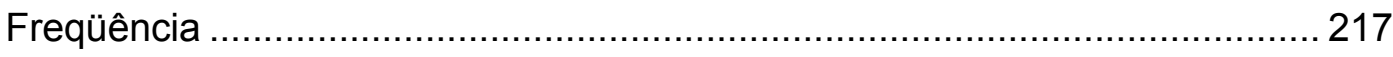

7.1 Mecanismo de Realocação de Energia [MAE, 2003d] ...................................217

7.1.1 Alocação da Energia Assegurada.........................................................219

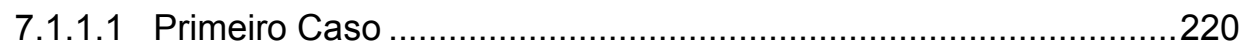

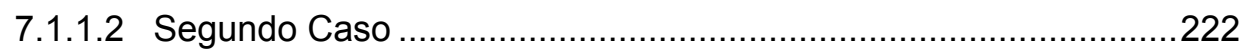

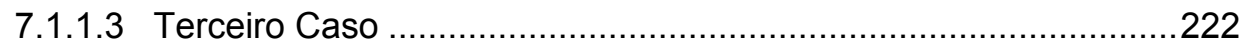

7.1.2 Alocação da Energia Assegurada entre Sub-Mercados ............................226

7.1.3 Alocação da Energia Secundária.........................................................227

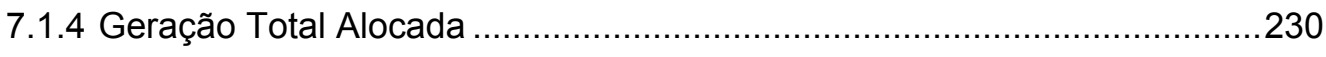

7.1.5 Custo da Compensação da Geração..................................................231

7.2 Influência do MRE na Remuneração dos Agentes Fornecedores .......................233

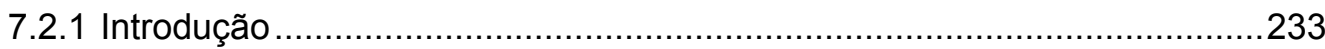

7.2.2 Aspectos Financeiros Relacionados à Participação no MRE .....................236

7.2.3 Recuperação de Custos Não Havendo Déficit de Geração.........................238

7.2.3.1 Recuperação dos Custos de Compensação da Geração..............238

7.2.3.2 Recuperação do Custo de Oportunidade ...................................239

7.2.4 Recuperação de Custos no Caso de Déficit de Geração ...........................240

8 Conclusões e Trabalhos Futuros ......................................................... 243 


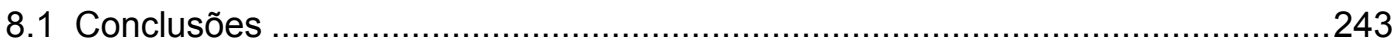

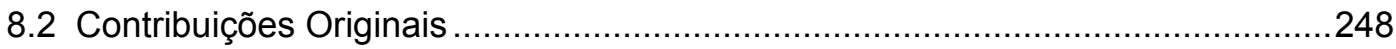

8.3 Sugestões para o Desenvolvimento de Trabalhos Futuros …...........................250

9 Referências Bibliográficas e Bibliografia .................................................. 253

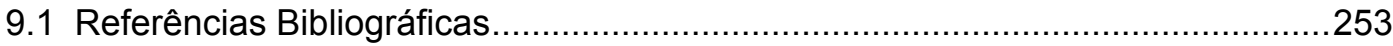

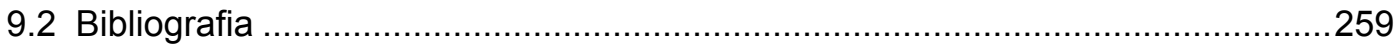

A Reservas de Potência ......................................................................... 263

Lista de Abreviaturas, Siglas, Acrônimos e Variáveis ..............................................263

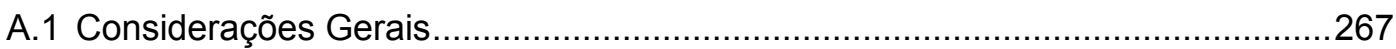

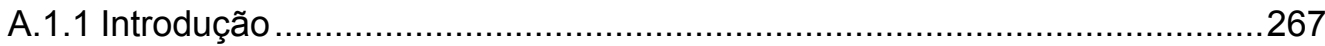

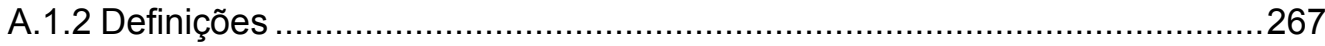

A.1.3 Considerações sobre a Quantificação e a Alocação das Reservas de ............

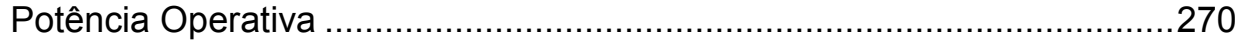

A.1.4 Considerações sobre a Utilização das Reservas Operativas ......................272

A.1.4.1 Erro de Controle de Área .......................................................272

A.1.4.2 Tempo de Disponibilização das Reserva e Taxas de Rampa ......273

A.2 Reservas de Potência Operativa no Brasil .........................................................278

A.2.1 Definição das Parcelas da Reserva de Potência Operativa ........................278

A.2.2 Quantificação das Parcelas da Reserva de Potência Operativa ................278

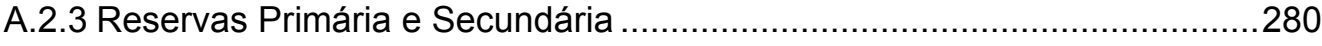

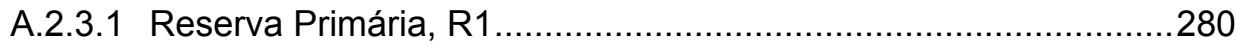

A.2.3.2 Reserva Secundária, R2 ...................................................281

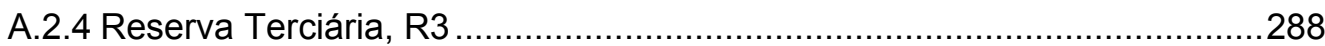

A.2.5 Considerações Sobre o Cálculo Probabilístico de R3 ...............................289

A.2.6 A Revisão 02 do Módulo 10 dos Procedimentos de Rede do ONS.............290

A.3 Referências Bibliográficas e Bibliografia ...........................................................292

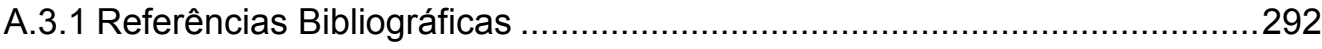

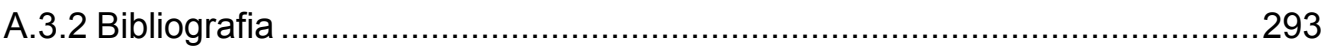

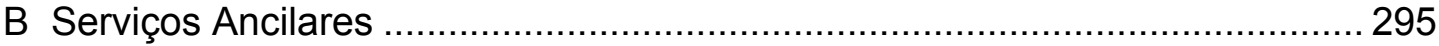

Lista de Abreviaturas, Siglas, Acrônimos e Variáveis .................................. 295

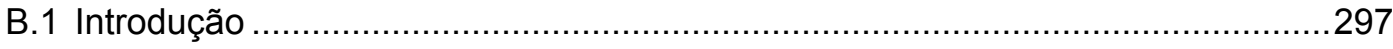

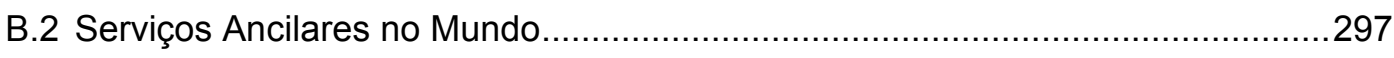

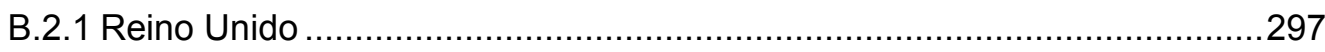

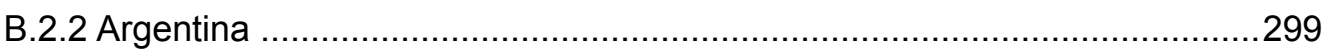




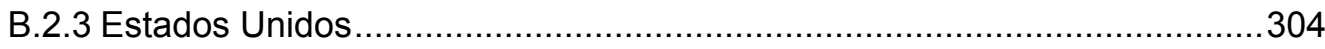

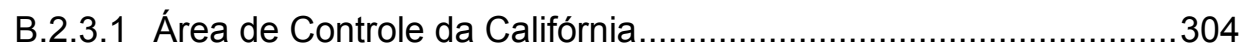

B.2.3.2 Área de Controle de New York ..................................................306

B.2.3.3 Área de Controle de New England ..........................................307

B.2.3.4 Outros Padrões Americanos de Quantificação .............................309

B.2.3.5 Comercialização e Remuneração .............................................310

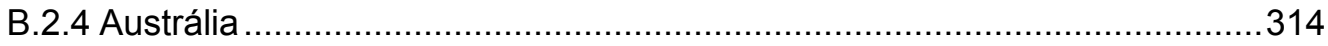

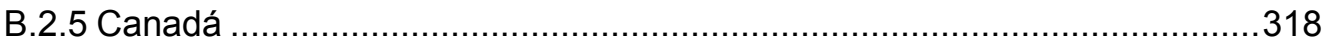

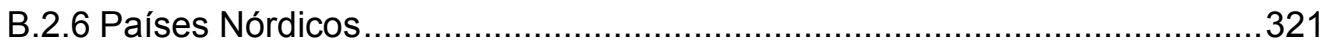

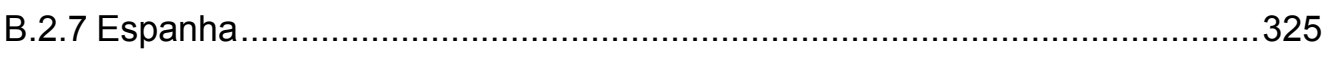

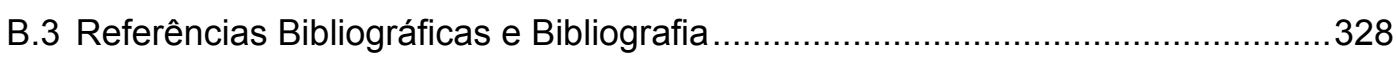

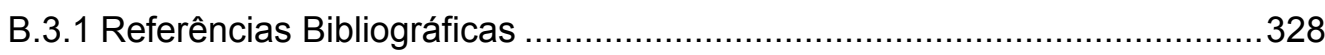

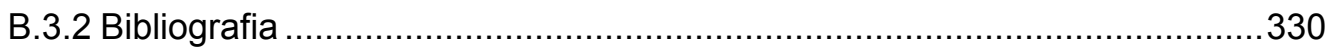

C Metodologias para o Cálculo do Encargo de Capacidade no Brasil............... 331

Lista de Abreviaturas, Siglas, Acrônimos e Variáveis ................................... 331

C.1 Metodologia Proposta no Processo de Reestruturação.........................................333

C.2 Metodologia Proposta pelo Comitê de Revitalização do Modelo do Setor Elétrico ...

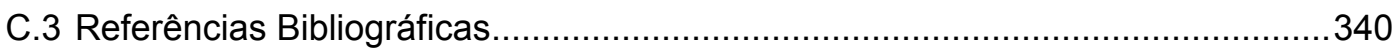




\section{Lista de Tabelas}

Tabela 2.1 Encargos de Capacidade Determinados pelo Estudo do Centro de Pesquisa

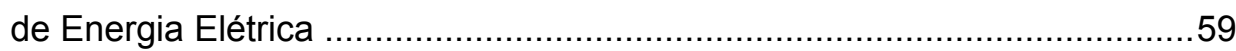

Tabela 2.2 Demais Resultados do Estudo - Metodologia Custo x Benefício....................60

Tabela 3.1 Usina Hidrelétrica: Dados e Parâmetros para o Cálculo dos Custos de Geração .91

Tabela 3.2 Usina Termelétrica: Dados e Parâmetros para o Cálculo dos Custos de

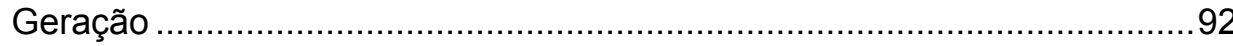

Tabela 3.3 Outras Tecnologias de Termo-Geração - Dados e Parâmetros Típicos.......93

Tabela 3.4 Componentes Fixos e Variáveis dos Custos de Geração ............................93

Tabela 3.5 Custos de Oportunidade Perdida em Não Gerar - Usinas 'Novas' ..............101

Tabela 3.6 Custos de Oportunidade Perdida em Não Gerar - Usinas 'Velhas' .............102

Tabela 3.7 Custos de Oportunidade Perdida em Não Gerar - Usinas 'Velhas', com Retorno sobre o Capital ..........................................................................102

Tabela 3.8 Preços dos Contratos Iniciais por Região................................................104

Tabela 3.9 Custos de Oportunidade em Relação aos Contratos Iniciais .....................104

Tabela 3.10 Potência Absorvida na Operação como Compensador Síncrono ...............116

Tabela 3.11 Custos de Partida e Operação em Vazio de Usinas Térmicas....................121

Tabela 5.1 Classes de Estatísticas de Falha Utilizadas pelo Ferramenta Computacional

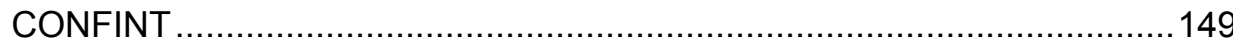

Tabela 5.2 Capacidades de Geração das Configurações do Sistema - MW................158

Tabela 5.3 Cargas Próprias Diárias do Sistema - MW ............................................. 158

Tabela 5.4 Curvas de Carga Diárias das Áreas Sul e Sudeste - 03 Patamares ..........159

Tabela 5.5 Valores Diários Observados da LOLP por Patamar de Carga - \% ............. 160

Tabela 5.6 Benefícios Incrementais Diários por Patamar de Carga - pu.......................160

Tabela 5.7 Remunerações Horárias por Patamar de Carga - Unidade Hidráulica $\mathrm{R} \$ / \mathrm{MWh}$.

Tabela 5.8 Remunerações Horárias por Patamar de Carga - Unidade Térmica $\mathrm{R} \$ / \mathrm{MWh}$. 161

Tabela 5.9 Programação de Reservas da Unidade Hidráulica - MWh ..........................162

Tabela 5.10 Programação de Reservas da Unidade Térmica - MWh ............................163

Tabela 5.11 Remunerações Totais Horárias - Unidade Hidráulica - R\$ ......................... 164

Tabela 5.12 Remunerações Totais Horárias - Unidade Térmica - R\$............................ 165 
Tabela 5.13 Remunerações Totais Diárias - Contabilização Final da Remuneração - R \$...

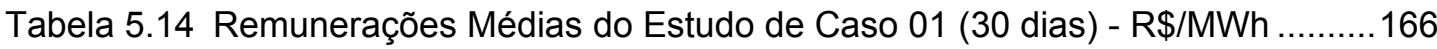

Tabela 5.15 Custos das Reservas de Confiabilidade e da Regulação de Freqüência $\mathrm{R} \$ / \mathrm{MWh}$ 166

Tabela 6.1 Fatores de Eficiência Horária da Regulação Secundária de Freqüência ...183

Tabela 6.2 Percentuais Horários de Regulação 'Para Cima'..... 191

Tabela 6.3 Percentuais Médios de Reserva para Regulação de Freqüência Observados

Tabela 6.4 Benefício Incremental do Serviço de Regulação de Freqüência - pu ..........194

Tabela 6.5 Remuneração Horária Básica do Serviço de Regulação de Freqüência - R\$..

Tabela 6.6 Remuneração Horária Final do Serviço de Regulação de Freqüência - R \$.....

196

Tabela 6.7 Valores Máximos da Remuneração Horária Básica do Serviço de Regulação de Freqüência - R \$ 197

Tabela 6.8 Remuneração Final da Regulação de Freqüência - Unidade Hidráulica....198

Tabela 6.9 Remunerações Unitárias pelo Benefício da Reserva para Confiabilidade Unidade Hidráulica 199

Tabela 6.10 Remunerações Unitárias pelo Benefício da Reserva para Confiabilidade Unidade Térmica 200

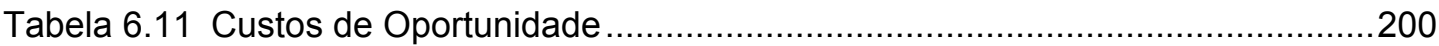

Tabela 6.12 Remunerações da Unidade Hidráulica - Caso 03.a...................................202

Tabela 6.13 Remunerações da Unidade Hidráulica - Caso 03.b...................................202

Tabela 6.14 Remunerações da Unidade Hidráulica - Caso 03.c..................................203

Tabela 6.15 Remunerações da Unidade Hidráulica - Caso 03.d..................................204

Tabela 6.16 Remunerações da Unidade Hidráulica - Caso 03.e.....................................205

Tabela 6.17 Remunerações da Unidade Hidráulica - Caso 03.f....................................206

Tabela 6.18 Remunerações da Unidade Térmica - Caso 03.j.....................................207

Tabela 6.19 Remunerações da Unidade Térmica - Caso 03.k ....................................208

Tabela 6.20 Remunerações da Unidade Térmica - Caso 03.I......................................209

Tabela 6.21 Remunerações da Unidade Térmica - Caso 03.m...................................210

Tabela 6.22 Remunerações da Unidade Térmica - Caso 03.n......................................211

Tabela 6.23 Remuneração Unitária do Benefício da Regulação de Freqüência.............212

Tabela 6.24 Remuneração da Regulação de Freqüência - Unidade Hidráulica 'Velha' .213

Tabela 6.25 Remuneração da Regulação de Freqüência - Unidade Hidráulica 'Nova' ..214 
Tabela 6.26 Remuneração da Regulação de Freqüência - Unidade Hidráulica Considerando os Preços dos Contratos Iniciais. ....................................215

Tabela 6.27 Remuneração da Regulação de Freqüência - Unidade Hidráulica..............215

Tabela A.1 Quantificação das Reservas Operativas em Diversas Empresas ..............271

Tabela A.2 Faixas de Variação do $\operatorname{ECA}\left(L_{10}\right)$ em Função da Dimensão da Área de Controle - Eastern Connection - EUA .......................................................276 


\section{Lista de Figuras}

Figura 2.1 Determinação do Encargo de Capacidade e da Capacidade Requerida .......59

Figura 2.2 Reservas de Planejamento e Operativas - Relacionamento e Remuneração....

Figura 3.1 Curvas Típicas de Eficiência das Turbinas Hidráulicas [Skrotzki, 1960] ......118

Figura 5.1 Curva Parcial LOLP x RES de um Sistema Elétrico ...................................143

Figura 5.2 Curvas LOLP x RES - Diversas Unidades Geradoras ............................... 145

Figura 5.3 Curvas LOLP x RES - Diversas Unidades Geradoras ............................... 146

Figura 5.4 Forma Típica da Curva LOLP x RES Completa....................................... 153

Figura 5.5 Forma Típica da Curva BI_RES x RES ................................................. 154

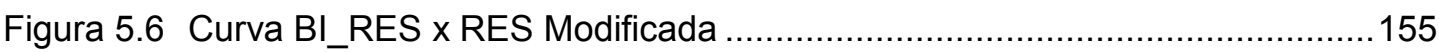

Figura 6.1 Valores Filtrados da Freqüência - Filtro de 6 Minutos, Uma Aplicação ........184

Figura 6.2 Valores Filtrados da Freqüência - Filtro de 6 Minutos, Duas Aplicações......184

Figura 6.3 Formas Típicas das Curvas FER x rr e BI_SR x rr .....................................187

Figura 6.4 Leituras de Freqüência - Após Dupla Aplicação do Filtro de 6 Minutos .......190

Figura 6.5 Percentuais Horários de Regulação ‘Para Cima' e 'Para Baixo' .................... 192

Figura 6.6 Curvas do Fator de Eficiência Horária x Reserva Percentual ....................... 193

Figura 6.7 Curvas do Benefício Incremental Horário x Reserva Percentual...................193

Figura 7.1 Realocação da Energia Assegurada - Primeiro Caso ..................................221

Figura 7.2 Realocação da Energia Assegurada - Segundo caso …..........................224

Figura 7.3 Realocação da Energia Assegurada - Terceiro Caso ..................................225

Figura 7.4 Realocação da Energia Secundária - Segundo Caso..................................229

Figura A.1 Demanda Prevista na Hora j de um Determinado Dia ................................283

Figura A.2 Demanda Observada na Hora j de um Determinado Dia .............................283

Figura A.3 Demanda Observada e Demanda de Ponta na Hora j. ...............................285

Figura A.4 Demanda Observada em Certas Horas com Ponta Dentro da Ponta na

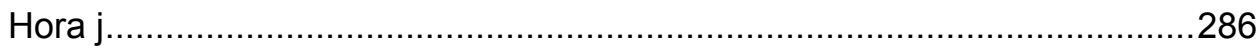




\title{
Lista de Abreviaturas, Siglas, Acrônimos e Variáveis
}

\author{
a - índice realativo às áreas de controle \\ $\mathrm{ad}_{\mathrm{i}}$ - fator de abertura do disjuntor da unidade geradora i, para operação \\ em vazio, não sincronizada \\ AGC - automatic generation control \\ $A L \_A S S_{p j}$ - montante de energia assegurada recebida pela usina $p$, na hora $j$, \\ sem considerar o excedente de Itaipu - MWh \\ $A L \_G_{p j}$ - geração total alocada a uma usina qualquer do MRE, pertencente a \\ um submercado s, em um período de comercialização j - MWh \\ ANEEL - Agência Nacional de Energia Elétrica \\ $\overline{\mathrm{ASS} \_}{ }_{\mathrm{p}}$ - energia assegurada média da usina $\mathrm{p}$, no ano $\mathrm{f}-\mathrm{MWh}$ \\ ASS $1_{p j}$ - energia assegurada da usina $p$, na hora $\mathrm{j}$ - MWh \\ ASS_2 ${ }_{\mathrm{pj}}$ energia assegurada ajustada da usina $\mathrm{p}$, na hora j - MWh \\ $B$ - ajuste de bias de uma área de controle \\ $B E F \_S R_{i j}$ - remuneração final da unidade geradora i, pelo benefício \\ proporcionado por sua participação no serviço de regulação de \\ freqüência, na hora j - \$ \\ $B E F \_S R_{i j d m}^{(L)}$ - remuneração final da unidade geradora $i$, pelo serviço de regulação \\ de freqüência, para o patamar de carga leve, numa hora j em um dia \\ $\mathrm{d}$, mês $\mathrm{m}$ - \$ \\ $B E F \_S R_{j d m}^{(M)}$ - remuneração final da unidade geradora i, pelo serviço de regulação \\ de freqüência, para o patamar de carga média, numa hora j em um \\ dia d, mês $\mathrm{m}$ - \$ \\ $B E F \_S R_{j d m}^{(P)}$ - remuneração final da unidade geradora i, pelo serviço de regulação \\ de freqüência, para o patamar de carga pesada, numa hora j em um \\ dia d, mês $m$ - \$ \\ Bef_SR $\mathrm{ij}_{\mathrm{ij}}$ - remuneração final unitária da unidade geradora i, pelo benefício \\ proporcionado por sua participação no serviço de regulação de \\ freqüência, na hora j - \$/MWh \\ $\mathrm{BE} \_R C f_{i j}$ - remuneração pelo benefício de uma certa quantidade de reserva \\ para confiabilidade, fornecida pela unidade geradora i, na hora j - \$ \\ $B e \_R C f_{i j}$ - remuneração unitária da unidade geradora i, pelo benefício da sua \\ reserva para confiabilidade, na hora $\mathrm{j}$ - $\$ / \mathrm{MWh}$ \\ $B e \_R C f_{i j d m}^{(L)}$ - remuneração unitária pelo benefício incremental da reserva de \\ confiabilidade da unidade geradora i, no patamar de carga leve, para \\ a hora j, do dia d, mês $m$ - \$/MWh \\ $B e \_R C f_{i j d m}^{(M)}$ - remuneração unitária pelo benefício incremental da reserva de \\ confiabilidade da unidade geradora i, no patamar de carga média, \\ para a hora j, do dia d, mês $\mathrm{m}$ - \$/MWh \\ $B e \_R C f_{i j d m}^{(P)}$ - remuneração unitária pelo benefício incremental da reserva de \\ confiabilidade da unidade geradora $\mathrm{i}$, no patamar de carga pesada, \\ para a hora j, do dia d, mês $\mathrm{m}$ - \$/MWh
}


$B e_{-} R_{i j d m}^{(P, M, L)}$ - remuneração unitária pelo benefício incremental da reserva de $\mathrm{m}$, em qualquer patamar de carga - $\$ / \mathrm{MWh}$

BE_Regidm - remuneração da unidade geradora i pelo benefício da regulação de freqüência, no dia $d$, mês $m$ - \$

$B E \_R e g_{i j}$ - remuneração da unidade geradora i pelo benefício da regulação de freqüência, na hora j - \$

BE_Regim - remuneração da unidade geradora i pelo benefício da regulação de freqüência, no mês $m$ - \$

Be_Reg $g_{i j}$ - remuneração unitária da unidade geradora i pelo benefício da regulação de freqüência, na hora j - \$/MWh

Be_Reg $g_{i j d m}^{(L)}$ - remuneração unitária da unidade geradora i pelo benefício da regulação de freqüência, no patamar de carga leve na hora j, no dia $\mathrm{d}$, mês $\mathrm{m}$ - \$/MWh

$B e \_\operatorname{Reg}_{\mathrm{ijdm}}^{(\mathrm{M})}$ - remuneração unitária da unidade geradora i pelo benefício da regulação de freqüência, no paríodor de carga média na hora j, no dia d, mês $m$ - \$/MWh

$\mathrm{Be} \_\operatorname{Reg}_{\mathrm{ijdm}}^{(\mathrm{P})}$ - remuneração unitária da unidade geradora i pelo benefício da regulação de freqüência, no paríodo de carga pesada na hora j, no dia d, mês $\mathrm{m}$ - \$/MWh

$B e \_\operatorname{Reg}_{\mathrm{ijdm}}^{(P, M, L)}$ - remuneração unitária da unidade geradora i pelo benefício da regulação de freqüência, em qualquer patamar de carga na hora j, no dia d, mês $\mathrm{m}$ - \$/MWh

$B E \_\operatorname{Res}_{\mathrm{ij}}$ - remuneração pelo benefício de uma certa quantidade de reserva operativa, fornecida pela unidade geradora i, na hora j - \$

BE_Res $s_{i m}$ - remuneração pelo benefício da reserva operativa fornecida pela unidade geradora $\mathrm{i}$, no mês $\mathrm{m}$ - \$

$B E \_R e s_{p j}$ - remuneração total pelo benefício de uma certa quantidade de reserva, fornecida pela usina $p$, na hora $\mathrm{j}$ - $\$$

$B E \_R g_{i j}$ - remuneração pelo benefício de uma certa quantidade de reserva para regulação, fornecida pela unidade geradora i, na hora j - \$

$B e \_R g_{i j}$ - remuneração unitária da unidade geradora $i$, pelo benefício de sua reserva para regulação de freqüência, na hora j - $\$ / M W h$

$B e_{-} R R g\left(\mathrm{ijdm}_{\mathrm{j}}^{(\mathrm{L})}\right.$ - remuneração unitária pelo benefício da reserva de regulação da unidade geradora i, no patamar de carga leve, para a hora j, do dia d, do mês $\mathrm{m}$ - \$/MWh

$B e \_R R g\left(\begin{array}{ll}(\mathrm{M}) \\ \mathrm{j} d m\end{array}\right.$ unidade geradora i, no patamar de carga média, para a hora j, do dia $\mathrm{d}$, do mês $\mathrm{m}$ - \$/MWh

$B e \_R R g\left(\begin{array}{l}(\mathrm{P}) \\ \mathrm{j} d m\end{array}\right.$ - remuneração unitária pelo benefício da reserva de regulação da unidade geradora i, no patamar de carga pesada, para a hora j, do dia d, do mês $\mathrm{m}$ - $\$ / \mathrm{MWh}$

$B E \_S R_{i j}$ - remuneração básica da unidade geradora $i$, pelo benefício de sua participação no serviço de regulação, na hora j - \$

$B E \_S R_{i j d m}^{(L)}$ - remuneração básica da unidade geradora $i$, pelo serviço de regulação de freqüência, para o patamar de carga leve, numa hora j em um dia d, mês $m$ - \$ 
$\mathrm{BE} \_\mathrm{SR}_{\mathrm{jdm}}^{(\mathrm{M})}$ - remuneração básica da unidade geradora $\mathrm{i}$, pelo serviço de j em um dia d, mês $m$ - \$

$B E \_S R_{j d m}^{(P)}$ - remuneração básica da unidade geradora $i$, pelo serviço de regulação de freqüência, para o patamar de carga pesada, numa hora j em um dia d, mês $m$ - \$

$\mathrm{BI} \_R C f_{i j}$ - benefício incremental da reserva operativa para confiabilidade da unidade geradora i, na hora $\mathrm{j}$ - pu

$\mathrm{BI}_{-} \mathrm{RCf}_{\mathrm{icdm}}^{(\mathrm{L})}$ - benefício incremental da reserva de confiabilidade da unidade geradora i, no patamar de carga leve, para a configuração de geração c, dia d e mês $m$ - pu

$\mathrm{BI} \mathrm{RCf}_{\mathrm{idm}}^{(\mathrm{L})}$ - benefício incremental da reserva de confiabilidade da unidade geradora i, no patamar de carga leve, no dia d e mês $m$, com configuração de geração única - pu

$\mathrm{BI} \mathrm{RCF}_{\mathrm{icdm}}^{(\mathrm{M})}$ - benefício incremental da reserva de confiabilidade da unidade geradora i, no patamar de carga média, para a configuração de geração c, dia d e mês $m$ - pu

BI_RCf $f_{\text {idm }}^{(M)}$ - benefício incremental da reserva de confiabilidade da unidade geradora i, no patamar de carga média, no dia d e mês $\mathrm{m}$, com configuração de geração única - pu

$\mathrm{BI} \mathrm{RCF}_{\mathrm{icdm}}^{(\mathrm{P})}$ - benefício incremental da reserva de confiabilidade da unidade geradora i, no patamar de carga pesada, para a configuração de geração $\mathrm{c}$, dia d e mês $\mathrm{m}$ - pu

$B I$ RCf $(\mathrm{P})$ - benefício incremental da reserva de confiabilidade da unidade geradora i, no patamar de carga pesada, no dia $d$ e mês $m$, com configuração de geração única - pu

$\mathrm{BI}$ _RES $\mathrm{ij}_{\mathrm{j}}$ - benefício incremental da reserva operativa da unidade geradora i, na hora j - pu

$\mathrm{BI}_{\text {_ }} \mathrm{RES}_{\text {icdm }}^{\text {máx }}$ - valor máximo do benefício incremental da reserva operativa da unidade geradora i, na configuração de geração c, no dia d e mês m $\mathrm{pu}$

BI_RRg $\underset{\text { icdm }}{(\mathrm{L})}$ - benefício incremental da reserva de regulação da unidade geradora i, observado no patamar de carga leve, na configuração de geração c, no dia $d$ e mês $m$ - pu

$\mathrm{BI} \_R R g \underset{i c d m}{(\mathrm{M})}$ - benefício incremental da reserva de regulação da unidade geradora i, observado no patamar de carga média, para a configuração de geração c, dia d e mês m - pu

BI_RRg $\underset{\text { icdm }}{(P)}$ - benefício incremental da reserva de regulação da unidade geradora i, observado no patamar de carga pesada, para a configuração de geração c, dia d e mês $m$ - pu

$\mathrm{BI} \_S R_{\mathrm{ij}}$ - benefício incremental do serviço de regulação de freqüência, de uma unidade geradora i, em uma hora j - pu

$\mathrm{BI} \mathrm{SR}_{\mathrm{jdm}}^{(\mathrm{L})}$ - benefício incremental do serviço de regulação de freqüência, para o patamar de carga leve, numa hora j em um dia d, mês $\mathrm{m}$ - pu

$B I \_S R_{j d m}^{(M)}$ - benefício incremental do serviço de regulação de freqüência, para o patamar de carga média, numa hora j em um dia d, mês $\mathrm{m}$ - pu

BI_SR $(\mathrm{P})$ - benefício incremental do serviço de regulação de freqüência, para o patamar de carga pesada, numa hora j em um dia d, mês $m$ - pu 
$\mathrm{CAd}_{\mathrm{pj}}$ - custo adicional de uma usina $\mathrm{p}$, participante do MRE, ao fornecer reservas girantes, em uma hora $\mathrm{j}$ - $\mathrm{R} \$$

CAG - controle automático da geração

Cag $_{\mathrm{ij}}$ - custo unitário do uso da água para uma unidade geradora i, na hora j - $\$ / \mathrm{m}^{3}$

CAMMESA - Compañia Administradora del Mercado Mayorista Eléctrico Argentina

CA_N $\mathrm{N}_{\mathrm{i}}$ - capacidade nominal de geração da unidade geradora i - MW

CA_N $N_{p}$ - capacidade nominal de geração da usina $p$ - MW

CAP - montante anual da capacidade a ser remunerada - GWh

CCC - conta de consumo de combustível

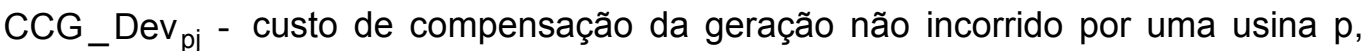
em uma hora j, devido ao ajuste da energia assegurada - $\mathrm{R} \$$

$\mathrm{CCOM}_{\mathrm{i}}$ - consumo horário de energia na compressão de ar da unidade geradora i, para rebaixamento do canal de fuga - MWh

CEMIG - Companhia Energética de Minas Gerais

CEPEL - Centro de Pesquisa de Energia Elétrica

CESP - Companhia Energética de São Paulo

Cf2a $a_{p}$ custos fixos anuais da usina $p$, com exceção dos custos de capital \$/MWano

CF_IN $\mathrm{N}_{\mathrm{p}}$ - custo fixo anual de investimento da usina $\mathrm{p}$ - \$/MWano

Cf_I $\mathrm{N}_{p}$ - custo fixo unitário de investimento da usina $\mathrm{p}-\$ / \mathrm{MWh}$

$\mathrm{Cf}_{\mathrm{pj}}$ - custo fixo unitário de produção da usina p na hora j - \$/MWh

$C F x_{p}$ - custo fixo da produção de uma certa quantidade de energia pela usina $\mathrm{p}-\$$

CGEY - Capgemini \& Young - Consulting, Technology, Outsourcing - EUA

$\mathrm{CGG}_{\mathrm{cadm}}$ - capacidade de geração disponível da área de controle a, na configuração de geração $c$, dia d e mês $\mathrm{m}$ - MW

$\mathrm{CGG}_{\mathrm{cdm}}$ - capacidade de geração disponível do sistema, na configuração de geração $\mathrm{c}$, dia d e mês $\mathrm{m}$ - MW

$\mathrm{CH}_{\mathrm{c}}$ - conjunto de horas da configuração de carga c

CHESF - Companhia Hidroelétrica do São Francisco

$\mathrm{C}_{\text {int }}$ - custo unitário da interrupção - Brasil - $\mathrm{R} \$ / M W h$

$\mathrm{Ci}_{\text {_ }} \operatorname{Res}_{\mathrm{ij}}$ custo unitário específico incorrido pela unidade geradora $\mathrm{i}$, na hora $\mathrm{j}$, relativo ao serviço de reservas operativas - \$/MWh

$\mathrm{Cl}_{\text {_Res }} \mathrm{R}_{\mathrm{pj}}$ - custo específico incorrido pela usina $\mathrm{p}$, na hora j, relativo ao serviço de reservas operativas, para um certa quantidade de reserva - $\$$

$\mathrm{Ci}_{-} \operatorname{Res}_{\mathrm{pj}}$ custo unitário específico incorrido pela usina $\mathrm{p}$, na hora j, relativo ao serviço de reservas operativas - \$/MWh

$\mathrm{CMOT}_{\mathrm{i}}$ - demanda da unidade geradora i para operar como motor síncrono MW

CMS - custo marginal de operação de curto prazo do sistema - \$/MWh

COEX - Comitê Executivo do Mercado Atacadista de Energia

COFINS - contribuição para o financiamento da seguridade social

COMAE - Conselho do Mercado Atacadista de Energia Elétrica 
ConAg $\mathrm{i}_{\mathrm{i}}$ - consumo unitário de água da unidade geradora $\mathrm{i}-\mathrm{m}^{3} / \mathrm{MWh}$

CONFINT - ferramenta computacional para Análise de Confiabilidade de Sistemas Hidrotermelétricos Interligados - CEPEL

$\mathrm{CO} \_\mathrm{Nre}_{\mathrm{pj}}$ - custo de oportunidade não recuperado por uma usina $\mathrm{p}$, em uma hora j, devido ao ajuste da energia assegurada - $R \$$

COPEL - Companhia Paranaense de Energia

CO_Res $s_{p j}$ - custo de oportunidade já recuperado por uma usina $p$, devido à transferência de energia assegurada para ela, referente a energia não gerada para ser mantida como reserva, na hora $\mathrm{j}$ - $\mathrm{R} \$$

COSR-SE - Centro Regional de Operação do Sistema - Sudeste

$\mathrm{COST}_{\mathrm{pm}}$ - custo a ser pago pela geração, em um mês $\mathrm{m}$ de apuração, para uma usina $p$ - R $\$$

$\mathrm{Cov}_{\mathrm{i}}$ - custo de operação em vazio da unidade geradora i - \$/h

$C P_{i}$ - custo de partida da unidade geradora i - \$/partida

$\mathrm{Cpe}_{\mathrm{p}}^{(1)}$ - custo unitário de produção da energia, da usina $\mathrm{p}$, para o fator de capacidade $\mathrm{FC}_{\mathrm{p}}-\$ / \mathrm{MWh}$

$\mathrm{Cpe}_{\mathrm{p}}^{(2)}$ - custo unitário de produção da energia, da usina $\mathrm{p}$, para o fator de capacidade $\mathrm{fC}_{\mathrm{p}}^{(2)}-\$ / \mathrm{MWh}$

$\left(\mathrm{Cpe}_{\mathrm{pj}}\right)^{\text {opmin }}$ - custo unitário de produção da usina $\mathrm{p}$, sob condições mínimas de geração, no período de comercialização j - \$/MWh

$C P E_{p}$ - custo total da produção de uma certa quantidade de energia pela usina $\mathrm{p}-\$$

$\mathrm{Cpe}_{\mathrm{pj}}$ - custo unitário da produção da usina p, na hora j - \$/MWh

CRMSE - Comitê de Revitalização do Modelo do Setor Elétrico

CSSL - contribuição social sobre o lucro líquido

$C T$ Res $s_{p j}$ - custo total da usina $p$, na hora $j$, relativo ao serviço de reservas operativas, para um certa quantidade de reserva - $\$$

CUSTO_MRE - custo variável da usina para trocas de energia no MRE - R $\$ / M W h$

$\mathrm{CV} 2_{\mathrm{p}}$ - somatório dos custos variáveis da usina $\mathrm{p}$, na produção de uma certa quantidade de energia, exceto o custo relativo ao consumo de combustível ou à compensação financeira pelo uso da água - \$

$C_{V A G}$ - custo variável da usina hidráulica $p$, relativo à compensação financeira pelo uso da água, na produção de uma certa quantidade de energia - \$

$\mathrm{CVCB}_{p}$ - custo variável da usina térmica $p$, relativo ao consumo de combustível, na produção de uma certa quantidade de energia - \$

$\mathrm{Cvcb}_{\mathrm{p}}$ - custo unitário do combustível da usina térmica $\mathrm{p}-\$ / \mathrm{MWh}$

$C v_{p j}$ - custo variável unitário de produção da usina p na hora j - \$/MWh

$\overline{\mathrm{CV}}_{\mathrm{p}}$ - custo variável unitário médio da usina $\mathrm{p}$, no ano $\mathrm{f}$ - $\$ / \mathrm{MWh}$

$C V r_{p}$ - custo variável da produção de uma certa quantidade de energia pela usina $\mathrm{p}-\$$

$\mathrm{d}$ - índice relativo aos dias

D - coeficiente de amortecimento de um sistema elétrico ou de uma área de controle 
$D_{\text {adm }}^{(L)}$ - nível de demanda referente ao patamar de carga leve, da área de controle a, no dia d, do mês $\mathrm{m}$ - pu

$D_{\text {adm }}^{(M)}$ - nível de demanda referente ao patamar de carga média, da área de controle a, no dia d, do mês $\mathrm{m}$ - pu

$D_{a d m}^{(P)}$ - nível de demanda referente ao patamar de carga pesada, da área de controle a, no dia d, do mês $\mathrm{m}$ - pu

DEF_Dsp $p_{\mathrm{pj}}$ - parcela do déficit de geração provocado pela otimização do despacho, da usina $\mathrm{p}$, na hora $\mathrm{j}$ - MWh

Deficit $_{\mathrm{sj}}$ - déficit total de geração do sub-mercado s na hora j - MWh

Deficit_1 $1_{\mathrm{pj}}$ - déficit após o ajuste de segundo estágio de alocação de energia, da usina $\mathrm{p}$, na hora $\mathrm{j}$ - MWh

DEF_Res $s_{\mathrm{pj}}$ - parcela do déficit de geração provocado pela manutenção de reservas operativas girantes, da usina $\mathrm{p}$, na hora $\mathrm{j}$ - MWh

DEF_Rg $1_{p j}$ - parcela do déficit de geração provocado pelas reservas operativas girantes para regulação primária, da usina $\mathrm{p}$, na hora $\mathrm{j}$ - MWh

$\mathrm{DEF} \_\mathrm{Rg} 2_{\mathrm{pj}}$ - parcela do déficit de geração provocado pelas reservas operativas girantes para regulação secundária, da usina $p$, na hora $\mathrm{j}$ - MWh

$\mathrm{DEF} \_\mathrm{RRg}_{\mathrm{pj}}$ - parcela do déficit de geração provocado pelas reservas operativas girantes para regulação da usina $p$, na hora $\mathrm{j}$ - MWh

DEF_Rtr ${ }_{\mathrm{pj}}$ - parcela do déficit de geração provocado por restrições de transmissão, da usina $p$, na hora j - MWh

DEF_RCf $f_{p j}$ - parcela do déficit de geração provocado pelas reservas operativas girantes para confiabilidade da usina $p$, na hora $\mathrm{j}$ - MWh

DIM_Ass ${ }_{\mathrm{pj}}$ - diminuição do valor da energia originalmente assegurada da usina $\mathrm{p}$ na hora j - MWh

DOE - U. S. Department of Energy - EUA

$\mathrm{DON}_{\mathrm{pm}}$ - geração alocada para outras usinas, pela usina $\mathrm{p}$, na hora $\mathrm{j}$ - $\mathrm{MWh}$

$D s_{d m}^{(L)}$ - nível de demanda do sistema, referente ao patamar de carga leve, no dia d, do mês $m$

$D s_{d m}^{(M)}$ - nível de demanda do sistema, referente ao patamar de carga média, no dia d, do mês $m$

$D s_{d m}^{(P)}$ - nível de demanda do sistema, referente ao patamar de carga pesada, no dia d, do mês $\mathrm{m}$

EA _ $1_{\mathrm{pj}}$ - ajuste de primeiro estágio de alocação de energia, da usina $p$, na hora j - MWh

$E A \_2 A S S_{p j}$ - ajuste de segundo estágio de alocação de energia relativo à energia assegurada da usina $\mathrm{p}$, na hora $\mathrm{j}$ - MWh

$E A \_2 S E C_{p j}$ - ajuste de segundo estágio de alocação de energia relativo à energia secundária, da usina $\mathrm{p}$, na hora $\mathrm{j}$ - MWh

EA_3ASS ${ }_{\mathrm{plj}}$ - energia assegurada de um sub-mercado doador I, alocada para uma usina p localizada em um sub-mercado $\mathrm{s}$, em um período de comercialização j (ajuste de terceiro estágio de alocação de energia relativo à energia assegurada) - $\mathrm{MWh}$

$E A \_3 S E C_{p l j}$ - ajuste de terceiro estágio de alocação de energia relativo à energia secundária, da usina $\mathrm{p}$, na hora $\mathrm{j}$ - MWh

EA_IMP ${ }_{\text {pj }}$ - alocação de energia 'importada' de outros sub-mercados I pela usina $\mathrm{p}$, num período de comercialização j - MWh

EC - encargo de capacidade anual - \$ 
ECA - erro de controle de área - MW

ELETROSUL - Centrais Elétricas do Sul do Brasil S.A.

ERM_Def ${ }_{p j}$ - déficit de geração da usina p em relação à sua energia assegurada, na hora j

ERM_P $P_{\mathrm{pj}}$ - ajuste total do MRE da usina p, em uma hora j - MWh

ERM_Surp ${ }_{\mathrm{pj}}$ superávit de geração da usina $\mathrm{p}$ em relação à sua energia assegurada, na hora j

$E R N_{s j}$ - energia remanescente, no sub-mercado s, na hora j, após a alocação da energia assegurada - MWh

EUA - Estados Unidos da América

$E X C_{p s j}$ - excedente de alocação para uma usina $p$, participante do MRE e pertencente à quota $\mathrm{g}$ de geração de Itaipu (do próprio submercado), na hora j - MWh

$E X C_{p l j}$ - excedente de alocação para uma usina $p$, participante do MRE e pertencente à quota $g$ de geração de Itaipu (de outros submercados), na hora j - MWh

$\mathrm{f}$ - índice relativo aos anos

$\mathrm{fcm}_{\mathrm{i}}$ - fator de consumo da unidade geradora i para operar como motor síncrono - \%

$\mathrm{fc}_{\mathrm{p}}$ - fator de capacidade anual da usina $\mathrm{p} \mathrm{-} \mathrm{\%}$

$\mathrm{FC}_{\mathrm{p}}$ - valor do fator de capacidade anual da usina $\mathrm{p}$ a ser mantido - \%

$\mathrm{fc}_{\mathrm{p}}^{(2)}$ - valor observado do fator de capacidade anual da usina $\mathrm{p}$, no ano $\mathrm{f}$ -

$\mathrm{FEI}_{\mathrm{t}}$ - fator de eficiência instantâneo da regulação de freqüência

$\overline{\mathrm{FEI}_{\mathrm{t}}}$ - valor médio do fator de eficiência instantâneo da regulação de freqüência em um dado intervalo de tempo - \%

$F E R_{j}^{(1)}$ - fator de eficiência horária da regulação de freqüência, antes da disponibilização de reservas operativas para regulação de freqüência pela unidade geradora i, na hora $\mathrm{j}$ - \%

$\mathrm{FER}_{\mathrm{j}}^{(2)}$ - fator de eficiência horária da regulação de freqüência, após a disponibilização de reservas operativas para regulação de freqüência pela unidade geradora i, na hora $\mathrm{j}$ - \%

FERC - Federal Energy Regulatory Commission - EUA

$F E R_{j}^{(k)}$ - fator de eficiência horária da regulação de freqüência após o k-ésimo acréscimo na reserva para regulação, na hora j, por uma certa unidade geradora - \%

$F E R_{j}^{(k-1)}$ - fator de eficiência horária da regulação de freqüência antes do késimo acréscimo na reserva para regulação, na hora j, por uma certa unidade geradora - \%

$F E^{(T)}$ - fator de eficiência em um intervalo de tempo $T$ - \%

$f_{n}$ - freqüência nominal do sistema $-\mathrm{Hz}$

$f_{t}$ - freqüência do sistema no instante $t-H z$

GERASUL - Centrais Elétricas do Sul do Brasil S. A.

$G_{-} M R E_{p j}$ - energia gerada pelas usina $p$, pertencente ao MRE, na hora j - MWh 
$\bar{G}_{p}$ - geração horária média da usina $p$ no ano $f-M W h$

$G_{p j}$ - geração média de uma usina $p$, em uma hora j - MW

GR - conjunto de unidades fornecedoras de reservas em cada configuração

$\mathrm{GSF}_{\mathrm{j}}$ - fator de ajuste da energia assegurada na hora j - pu

i - índice relativo às unidades geradoras

$\mathrm{IC}_{\mathrm{p}}$ - investimento de capital da usina $\mathrm{p}-\$ \mathrm{MW}$

IR - imposto de renda

IRPJ - imposto de renda da pessoa jurídica

j - índice relativo aos períodos de comercialização, definido como uma hora no Brasil

k - índice utilizado para contagem de iterações

$\mathrm{k}_{\text {PPAD }}$ - constante de ajuste, definida em função da necessidade de se incentivar a implantação de nova geração - Argentina

$\mathrm{L}$ - conjunto de horas do período de carga leve do dia d

$L c_{p j}$ - lucro unitário da usina $p$, na hora j - \$/MWh

$\mathrm{LC}_{\mathrm{pj}}$ - lucro da usina $\mathrm{p}$, na hora $\mathrm{j}$, na comercialização de uma certa quantidade de energia - \$

$L_{n}$ - demanda de ponta da carga $n$ (mensal ou anual) - MW

LOLP $_{\text {adm }}$ - valor de referência observado da probabilidade de perda de carga, da área de controle a, no dia d, mês $\mathrm{m}$ - \%

LOLP $_{\mathrm{adm}}^{(\mathrm{L})}$ - valor de referência observado da probabilidade de perda de carga, referente ao patamar de carga leve, da área de controle a, no dia d, do mês $m$ - \%

LOLP $_{\mathrm{adm}}^{(\mathrm{M})}$ - valor de referência observado da probabilidade de perda de carga, referente ao patamar de carga média, da área de controle a, no dia d, mês $\mathrm{m}$ - \%

$\operatorname{LOLP}_{a d m}^{(P)}$ - valor de referência observado da probabilidade de perda de carga, referente ao patamar de carga pesada, da área de controle a, no dia $\mathrm{d}$, mês $\mathrm{m}-\%$

$\operatorname{LOLP}^{(P, M, L)}$ valor mínimo da probabilidade de perda de carga, para definição do benefício incremental da reserva de confiabilidade, em qualquer patamar de carga - \%

$\operatorname{LOLPs}_{d m}^{(L)}$ - valor de referência observado da probabilidade de perda de carga do sistema, referente ao patamar de carga leve, no dia d, do mês $\mathrm{m}$ - \%

LOLPs $_{d m}^{(M)}$ - valor de referência observado da probabilidade de perda de carga do sistema, referente ao patamar de carga média, no dia d, do mês $\mathrm{m}$ $\%$

LOLPs $\mathrm{dm}_{\mathrm{P}}^{(\mathrm{P})}$ - valor de referência observado da probabilidade de perda de carga do sistema, referente ao patamar de carga pesada, no dia d, do mês $\mathrm{m}$ $\%$

LOLC - loss of load cost - US\$/MWh

LOLP - loss of load probability - \%

$\operatorname{LOLP}_{\mathrm{j}}^{(0)}$ - valor inicial da LOLP na hora $\mathrm{j}$, sendo a demanda da área de controle ou do sistema igual à disponibilidade de geração - \% 
$\operatorname{LOLP}_{j}^{(1)}$ - probabilidade de perda de carga do sistema, ou área de controle, em uma hora j, antes da disponibilização de reservas operativas para confiabilidade pela unidade geradora i - \%

$\operatorname{LOLP}_{j}^{(2)}$ - probabilidade de perda de carga do sistema ou área de controle, em uma hora j, após a disponibilização de reservas operativas para confiabilidade pela unidade geradora i - \%

$\operatorname{LOLP}_{j}^{(k)}$ - valor da LOLP na hora j, na k-ésima entrada da unidade geradora i $\%$

$\operatorname{LOLP}_{\mathrm{j}}^{(\mathrm{k}-1)}$ - valor da LOLP na hora $\mathrm{j}$, na (k-1)-ésima entrada da unidade geradora i - \%

$\mathrm{m}$ - índice relativo aos meses

$M$ - conjunto de horas do período de carga média do dia d

MAE - Mercado Atacadista de Energia - Brasil

MODCAR - ferramenta computacional para modulação de dados de carga CEPEL

MRE - mecanismo de realocação de energia

$\mathrm{n}$ - índice relativo às cargas

na - número de áreas de controle de um sistema

nc - número de configurações de geração em um dia

nd - número de dias em um certo mês

NETA - New Electricity Trading Arrangements -

$\mathrm{nf}_{\mathrm{j}}$ - número de valores da freqüência observados na hora $\mathrm{j}$

$n f^{(T)}$ - número de valores da freqüência observados no intervalo $T$

$\mathrm{NH} 2$ - ferramenta computacional para Análise de Confiabilidade Composta de Sistemas de Geração-Transmissão de Energia Elétrica - CEPEL

NYISO - New York Independent System Operator - EUA

O\&M - operação e manutenção

OFGEM - Office of Gas and Electricity Markets - UK

ONS - Operador Nacional do Sistema Elétrico - Brasil

$\mathrm{OPT}_{\mathrm{ij}}$ - custo de oportunidade da unidade geradora $\mathrm{i}$, na hora $\mathrm{j}$, relativo ao serviço de reservas operativas, para uma certa quantidade de reserva - \$

$\mathrm{OPT}_{\mathrm{pj}}$ - custo de oportunidade da usina $\mathrm{p}$, na hora j, relativo ao serviço de reservas operativas, para uma certa quantidade de reserva - $\$$

$\mathrm{Opt}_{\mathrm{pj}}$ - custo de oportunidade unitário da usina $\mathrm{p}$, na hora $\mathrm{j}$, relativo ao serviço de reservas operativas - \$/MWh

$\mathrm{Opt}_{\mathrm{pj}}^{(\mathrm{AC})}$ - custo de oportunidade unitário da usina $\mathrm{p}$, na hora $\mathrm{j}$, relativo ao serviço de reservas operativas, em ambiente competitivo - \$/MWh

$p$ - índice relativo às usinas ou agentes geradores

$P$ - conjunto de horas do período de carga pesada do dia d

PAY_ASS ${ }_{p j}$ - pagamento devido por geração, por uma usina p, em uma hora $j$, relativo apenas à energia assegurada que lhe é cedida - $R \$$

$P A Y_{p j}$ - pagamento devido por geração relativo a uma usina $p$, em uma hora $j$ - R\$ 
$P A Y_{p m}$ - pagamento devido por geração relativo a uma usina $p$, em um mês de apuração $m$ - R\$

PAY_Res $s_{p j}$ - custo a ser recuperado por uma usina $p$, devido ao pagamento efetuado no MRE, referente à energia não gerada para ser mantida como reserva operativa, como custos de compensação da geração, em uma hora $\mathrm{j}$ - $\mathrm{R} \$$

$P C$ - pagamento a um gerador pela capacidade despachada - \$/MWh

$\mathrm{PC}_{\mathrm{n}}$ - pagamento por capacidade feito pela carga $\mathrm{n}-\$$

$\mathrm{PC}_{\mathrm{TOT}}$ - total de pagamentos por capacidade recolhido (mensal ou anual) - \$

$P G_{t}$ - potência gerada no sistema no instante $t-M W$

$P G_{t-\Delta t}$ - potência gerada no sistema no instante $(t-\Delta t)-M W$

PIS - Programa de Integração Social

PJM - Pennsylvania/New Jersey/Maryland Interconnection - EUA

$\mathrm{PLM}_{\mathrm{j}}$ - preço de liquidação do mercado de energia na hora j - US $\$ / M W h$;

$P L M_{j}^{(R E S)}$ - preço de liquidação de um mercado de reservas na hora $j$ US $\$ / M W h$

Pr_ $\mathrm{Ci}_{\mathrm{ij}}$ - preço praticado nos contratos iniciais pela unidade geradora $\mathrm{i}$, na hora j - \$/MWh

Pot_1 $1_{1 j}$ - superávit líquido de geração de um sub-mercado doador I, em um período de comercialização j - MWh

Pot_1 $1_{\mathrm{sj}}$ - superávit líquido de geração do sub-mercado s na hora j - MWh

$\mathrm{Pot}_{\mathrm{sj}}$ - superávit total de geração do sub-mercado s na hora j - MWh

Pr_Ci $i_{p j}$ - preço praticado nos contratos iniciais pela usina $\mathrm{p}$, na hora $\mathrm{j}-\$ / \mathrm{MWh}$

$\operatorname{Pr} \mathrm{e}_{\mathrm{ij}}$ - preço de venda da energia da unidade geradora $\mathrm{i}$, na hora $\mathrm{j}$ - \$/MWh

Pr $e_{p j}$ - preço de venda da energia da usina $p$, na hora $\mathrm{j}$ - \$/MWh

$\mathrm{Pr}_{-} \mathrm{Mer}_{\mathrm{j}}$ - preço de mercado da energia na hora $\mathrm{j}$ - \$/MWh

q - índice relativo aos anos

$\mathrm{R}^{2}$ - fator de aderência de uma série de observações a uma curva a elas ajustada - \%

$R_{\text {_ }} B R_{p j}$ - receita bruta da usina $p$, na hora j, na comercialização de uma certa quantidade de energia - $\$$

$R B$ _Res $s_{p j}$ - parcela do benefício agregado à operação do sistema pela usina $p$, através do serviço de reservas operativas, traduzido financeiramente, para uma certa quantidade de reserva, na hora j - \$

$\mathrm{RCf}_{\mathrm{ij}}$ - reserva de confiabilidade fornecida pela unidade geradora $\mathrm{i}$, na hora $\mathrm{j}$ - valor médio - MW

$R C f_{j}$ - reserva de confiabilidade do sistema na hora j - valor médio - MW

$\mathrm{RCf}_{\mathrm{ijdm}}$ - reserva de confiabilidade fornecida pela unidade geradora $\mathrm{i}$, na hora $\mathrm{j}$, do dia d, mês $\mathrm{m}$ - valor médio - MW

$R C f_{i j}^{(k)}$ - reserva de confiabilidade na hora j, na k-ésima entrada da unidade geradora i como reserva, - MW

$\mathrm{REC}_{\mathrm{pm}}$ - geração alocada ou recebida de outras usinas, pela usina $p$, no mês $\mathrm{m}-\mathrm{MWh}$

REM - remuneração horária média da energia - \$/MWh 


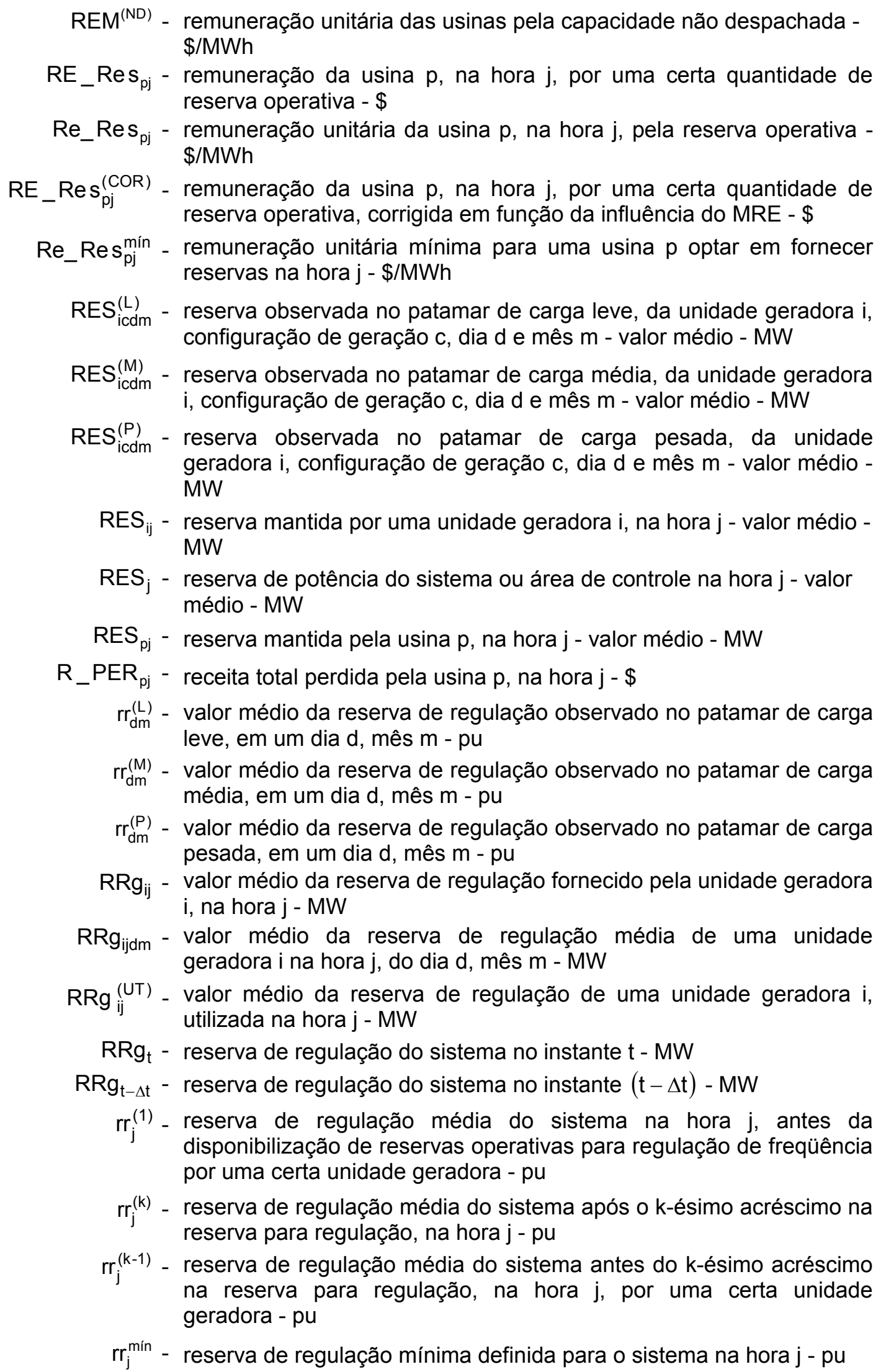


$\mathrm{rr}_{\mathrm{j}}^{(\mathrm{OBS})}$ reserva de regulação média do sistema, observada na hora j - pu

$\mathrm{rr}_{\mathrm{t}}$ - reserva de regulação do sistema no instante $\mathrm{t}$ - pu

$\mathrm{rr}_{\mathrm{t}}^{(\mathrm{R})}$ - reserva de regulação remanescente do sistema no instante $\mathrm{t}$ - $\mathrm{pu}$

$\mathrm{rr}_{\mathrm{t}}^{(\mathrm{UT})}$ - parcela da reserva de regulação que deveria ser utilizada pelos dispositivos de regulação por efeito do desvio de longa duração da freqüência $\Delta f p_{t}$, para retornar a freqüência ao seu valor nominal

$\mathrm{RRf}_{\mathrm{j}}^{\mathrm{min}}$ - reserva de regulação mínima definida para o sistema na hora j - MW

$R R f_{t}^{(R)}$ - reserva de regulação remanescente do sistema no instante $t$ - MW

$\mathrm{S}$ - estatismo equivalente do sistema - pu

SA - serviço ancilar

SASS $\_C_{p j}$ - direito à energia secundária proporcional à energia assegurada, da usina $\mathrm{p}$, na hora $\mathrm{j}$ - MWh

$\mathrm{SEC}_{\mathrm{j}}$ energia secundária total do sistema na hora $\mathrm{j}$ - MWh

TA - constante de tempo da água de uma turbina hidráulica - $s$

$\mathrm{TB}_{\mathrm{jdm}}$ - tempo em que a regulação é feita para cima na hora j, do dia d, mês $\mathrm{m}-\mathrm{pu}$

$\mathrm{TC}_{\mathrm{ij}}$ - tempo em que a regulação é feita para cima (freqüência baixa) pela unidade i, na hora j - pu

$\mathrm{TC}_{\mathrm{jdm}}$ - tempo em que a regulação é feita para cima na hora j, do dia d, mês $\mathrm{m}-\mathrm{pu}$

TM - constante de tempo de resposta do servo-motor do mecanismo de admissão, de uma turbina hidráulica - $s$

tr - taxa de retorno anual, ou taxa de juros anual prevista

TR - constante de tempo de um regulador de velocidade - $\mathrm{s}$

TS - constante de tempo de resposta de um sistema elétrico ou de uma área de controle - $\mathrm{s}$

ts - período em que uma unidade geradora permanece prestando o serviço de reservas operativas - $h$

TT - constantes de tempo de uma turbina térmica, que traduz a taxa de tomada de carga de cada tipo de conjunto turbina-gerador - s

TUST - taxa de uso do sistema de transmissão

$\mathrm{TVC}_{\mathrm{p}}$ - custo variável de MRE da usina $\mathrm{p}-\mathrm{R} \$ / \mathrm{MWh}$

VLL - value of lost load - US\$/MWh

VRF - custo associado à deficiência de regulação de freqüência no sistema durante um certo período de tempo

VU - vida útil da usina ou unidade geradora - anos

$\alpha_{\mathrm{pj}}$ - fator de participação da reserva operativa girante no déficit de geração em relação à energia assegurada de uma usina $p$, em uma hora j - \%

$\beta$ - característica natural de um sistema elétrico ou de uma área de controle

$\Delta \mathrm{Cpe}_{\mathrm{p}}$ - variação do custo de produção da energia, da usina $\mathrm{p}$, em função de uma variação no seu fator de capacidade - \$/MWh 
$\triangle F E R_{j}$ - variação do fator de eficiência horária da regulação de freqüência, devido à disponibilização de reservas operativas para regulação pela unidade geradora $\mathrm{i}$, na hora $\mathrm{j}$ - pu

$\Delta f^{\max }$ - variação da freqüência capaz de consumir toda a reserva de regulação existente, em um certo instante de tempo - $\mathrm{Hz}$

$\Delta f_{j}^{\text {máx }}$ - variação da freqüência capaz de consumir toda a reserva de regulação existente, em uma hora j - Hz

$\Delta f_{t}$ - desvio de freqüência remanescente em um certo instante de tempo $t$, após determinado período de tempo $\Delta \mathrm{t}$, de atuação da regulação de freqüência - $\mathrm{Hz}$

$\Delta f_{t-\Delta t}$ - desvio de freqüência remanescente no instante de tempo anterior ao instante $\mathrm{t}-\mathrm{Hz}$

$\Delta f \mathrm{p}_{\mathrm{t}}$ - componente lento dos desvios de freqüência em um instante $\mathrm{t}-\mathrm{Hz}$

$\Delta f_{t}^{(F)}$ - desvios instantâneos filtrados de freqüência - $\mathrm{Hz}$

$\overline{\left|\Delta \mathrm{fp}_{\mathrm{j}}\right|}$ - valor médio dos desvios lentos da freqüência em uma hora j - $\mathrm{Hz}$

$\left.\Delta \mathrm{LOLP}_{\mathrm{j}}\right|_{\mathrm{i}}$ - módulo da variação da probabilidade de perda de carga do sistema ou área de controle, em uma hora j, devido à disponibilização de reservas operativas para confiabilidade pela unidade geradora $\mathrm{i}$ - \%

$\Delta \mathrm{rr}_{\mathrm{j}}$ - acréscimo no valor da reserva para regulação, na hora j - pu

$\Delta R \operatorname{Rg}_{t}$ - variação da reserva de regulação devido a uma variação na freqüência - MW

$\Delta t$ - período de tempo de atuação da regulação de freqüência - $\mathrm{S}$

$\varepsilon_{\mathrm{T}}-$ erro de tempo - s/min

$\varepsilon_{\top}^{(+)}$- componente positiva do erro de tempo - s/min

$\varepsilon_{\top}^{(-)}$- componente negativa do erro de tempo - s/min

$\lambda_{i}$ - taxa de falhas da unidade geradora $\mathrm{i}$ - ocorrência/hora

$\mu_{\mathrm{i}}$ - taxa de reparos da unidade geradora i - ocorrência/hora

$\pi_{\mathrm{d}}$ - multiplicador simplex associado à restrição de atendimento a demanda em um sub-mercado

$\tau$ - ganho de um regulador de velocidade

$\sum_{\mathrm{f}}$ ASS ${ }_{-} 1_{\mathrm{pj}}-$ energia assegurada da usina $\mathrm{p}$ no ano $\mathrm{f}-\mathrm{MWh}$

$\sum_{1}$ - $\begin{aligned} & \text { somatório de todos os sub-mercados que cedem energia a uma } \\ & \text { usina } p \text {, em uma hora } j\end{aligned}$

$\sum_{p_{-} E R M}$ - somatório de todas as usinas $p$ participantes do MRE

$\sum$ - somatório de todas as usinas $\mathrm{p}$ participantes do MRE, pertencentes ps_ERM ao sub-mercado s

$\sum_{\mathrm{S}}$ - somatório de todos os sub-mercados

\$BASE - remuneração base unitária de potência - Argentina - peso/MW

\$PPAD - preço da potência posta à disposição - Argentina - peso/MW 